\title{
EchoGéo
}

42 | 2017

Varia

\section{Sous le signe du numérique}

Jean-Louis Chaléard

\section{(2) OpenEdition}

Journals

Édition électronique

URL : https://journals.openedition.org/echogeo/15176

DOI : 10.4000/echogeo.15176

ISSN : 1963-1197

\section{Éditeur}

Pôle de recherche pour l'organisation et la diffusion de l'information géographique (CNRS UMR 8586)

Référence électronique

Jean-Louis Chaléard, « Sous le signe du numérique », EchoGéo [En ligne], 42 | 2017, mis en ligne le 31 décembre 2017, consulté le 31 juillet 2021. URL : http://journals.openedition.org/echogeo/15176 ; DOI : https://doi.org/10.4000/echogeo.15176

Ce document a été généré automatiquement le 31 juillet 2021.

EchoGéo est mis à disposition selon les termes de la licence Creative Commons Attribution - Pas d'Utilisation Commerciale - Pas de Modification 4.0 International (CC BY-NC-ND) 


\title{
Sous le signe du numérique
}

\author{
Jean-Louis Chaléard
}

1 La nouvelle livraison d'Échogéo est particulièrement fournie, avec des textes dans la plupart des rubriques et un varia de six articles. On peut placer ce numéro sous le signe du numérique, objet de la table ronde de juin 2017, tenue à l'occasion des dix ans de notre revue, et dont on trouvera la retranscription dans le dossier Sur le métier. La question du numérique est à l'ordre du jour et la plupart des revues de géographie éditées sur papier se sont mises progressivement à ce format. La question a d'ailleurs été débattue lors de la table ronde sur les revues de géographie françaises au festival de géographie de Saint-Dié en octobre dernier. La forme même d'Échogéo, avec une rubrique en continu ou le recours à l'image, doit beaucoup au numérique.

2 La table ronde organisée en juin 2017 débordait largement la simple question des revues. Les cinq spécialistes conviés ont débattu à partir de leurs pratiques sur : «Le numérique: de nouvelles conditions du savoir?». Marie Pellen a présenté la plateforme revue.org, dont le succès et l'évolution avec le passage à Open Edition Journals attestent de l'importance qu'a pris internet aujourd'hui dans nos champs scientifiques, et a animé la séance. Stéphanie Delmotte nous a fait partager son expérience de chercheuse en sciences de l'information et de responsable d'un service de bibliothèque. Trois géographes nous ont fait part de leurs réflexions à partir de leur vécu professionnel: Matthieu Noucher qui travaille sur les nouvelles modalités de production, de diffusion et d'utilisation de l'information géographique numérique; Antonine Ribardière, responsable d'un master spécialisé dans le champ géomatique qui intègre une forte composante informatique; Malika Madelin dont les recherches portent principalement sur l'environnement avec un recours important à l'outil informatique. Les intervenants ont souligné le rôle croissant du numérique dans leur recherche (analyse de données, bibliographies...), la diffusion des savoirs (enseignement, publications...) et son influence sur leurs méthodes même d'investigation. Il et elles ont aussi souligné les limites et les dangers de l'outil, nous incitant à profiter des nouvelles opportunités offertes, mais avec vigilance et un vrai recul critique. 
3 Le corps du numéro est constitué par les sept articles du varia. Derrière le kaléidoscope des thèmes et des espaces, apparaissent quelques sujets dominants. D'abord, la question environnementale que l'on trouve, sous différentes formes, dans quatre textes. Annaig Oiry, constatant la faible prise en compte de la problématique du nucléaire dans le champ des études environnementales, s'interroge sur ce que pourraient être les contours d'une géographie du risque nucléaire. Elle insiste sur la nécessaire prise en compte de tous les acteurs intervenant dans la gestion ou la dénonciation du nucléaire et débouche sur une interrogation à propos de la mise en politique d'un niveau «acceptable» de risque. Solène Rey-Coquais examinant le projet hydro-électrique d'Alto Maipo, en région métropolitaine de Santiago du Chili, s'interroge sur les échelles d'intervention et de réflexion pour discuter de la justice environnementale. Elle montre la multiplicité des enjeux, les oublis des études d'impacts, les retards de la régulation environnementale publique et le rôle des mobilisations de la société civile contre le projet. Céline Raimbert et Nasser Rebai proposent une réflexion sur le rôle des collectifs publics pour le développement durable des territoires ruraux en Amérique latine. À partir de cas au Brésil et en Équateur, ils montrent comment les politiques publiques favorables à la constitution de ces collectifs permettent le renforcement des agricultures familiales, du ravitaillement des villes, de la protection de l'environnement. Échogéo avait déjà publié un article sur l'orpaillage au Niger. Le cas analysé ici, au Burkina Faso, souligne la fréquence de cette activité largement artisanale dans de nombreux pays sahélo-soudaniens. Dans son texte, Joseph Bohbot montre l'intérêt économique de l'orpaillage, mais aussi les problèmes qu'il engendre : entassement de migrants dans des campements de fortune, sans infrastructures sanitaires et sociales, pollutions liées à l'extraction de l'or, même si se développent (encore timidement) de nouvelles méthodes plus respectueuses des populations et de l'environnement.

Un second champ important abordé est celui du social. Il est déjà présent, comme nous venons de le voir, dans les articles qui s'intéressent aussi à des questions d'environnement, que ce soit à propos du risque nucléaire (et sa contestation), de la justice environnementale, des collectifs d'agriculteurs familiaux ou des conditions de travail et de vie des orpailleurs au Burkina Faso. Deux textes traitent de questions douloureuses à travers des problèmes liés à la migration. Laurence Pillant s'intéresse aux lieux d'enfermement des migrants à la frontière orientale de la Grèce. Entre enfermement, répression, déplacements forcés et incertitude sur leur sort, l'auteure montre que les migrants vivent entre l'intérieur des lieux d'enfermement et les réseaux de lieux dans lesquels ils sont pris. Pour Sarah Przybyl l'arrivée croissante en France de mineurs non accompagnés bouscule les logiques de gestion territoriale de l'enfance. Elle met en évidence l'enjeu géographique que représente leur répartition très hétérogène sur le territoire français et les problèmes que cela pose. Les frontières sont devenues poreuses entre la protection de l'enfance (qui relève du département) et le contrôle des flux migratoires (de compétence étatique), ce dernier prenant le pas, en dépit des contestations et de critiques venant de différents horizons. Le dernier texte, d'Alexandre Grondeau et Manon Boulpicante, renvoie à des interrogations sociales et environnementales, tout en étant centré surtout sur les nouvelles politiques municipales associées au privé et sur les projets de rénovation urbaine dans le cadre de la concurrence que se livrent les métropoles internationales entre elles. Alexandre Grondeau et Manon Boulpicante montrent que l'adaptation à la mondialisation et l'inquiétude sécuritaire ont eu raison des revendications d'un « droit 
à la ville» favorable à la création d'un territoire alternatif dans le quartier de Christiania à Copenhague.

Dans tous ces articles bien sûr d'autres thèmes s'entremêlent comme celui des politiques publiques dont l'importance est relevée par la plupart des auteurs. La réflexion sur les lieux ou les territoires, les questions d'échelles sont souvent présentes et nous interpellent sur les méthodes d'approche et d'analyse de la géographie pour tous ces sujets.

6 Les articles des autres rubriques sont également variés et permettent d'approcher différents thèmes ou différentes façons de faire de la géographie. Dans la rubrique Sur l'écrit, Sophie Didier, s'appuyant sur quelques ouvrages à succès, étudie la « fiction spéculative » sud-africaine, une forme de littérature de genre au service d'une critique de la société. L'auteure montre la rupture à bien des égards de cette littérature par rapport aux anciennes formes de production littéraire blanche engagée contre l'apartheid mais aussi la difficulté à s'en libérer totalement.

7 On reste en Afrique avec la rubrique Sur l'image consacrée au fleuve Sénégal, bel exemple de relations entre un cours d'eau et les territoires qu'il traverse. Laurent Bruckmann montre que le fleuve Sénégal a été tour à tour intégré puis exclu des grands ensembles géographiques : de la zone d'échanges mondialisée à l'époque de la traite, à la voie de communication marginalisée par la route plus récemment... Actuellement les logiques d'intégration par le bas des sociétés transfrontalières et les usages locaux de l'eau par les agriculteurs s'opposent aux logiques et aux projets tant politiques qu'économiques des États riverains. Le texte s'appuie sur de nombreux documents, cartes et photographies. Il confirme, comme nous l'avons relevé dans un précédent numéro, qu'un des intérêts d'une revue numérique est d'autoriser un large recours à l'image. Il convient d'en profiter... 